\title{
Role of direct immunofluorescence on outer root sheath of hair follicle in monitoring disease activity of pemphigus vulgaris
}

\author{
Nessa Mst Sayedatun ${ }^{1}$, Nurul Kabir A², Barua AR ${ }^{2}$, Paul Harasit Kumar ${ }^{3}$ \\ ${ }^{1}$ Department of Pathology, Northern International Medical College \& Hospital, Dhaka, Bangladesh \\ ${ }^{2}$ Department of Pathology, Bangabandhu Sheikh Mujib Medical University, Dhaka, Bangladesh \\ ${ }^{3}$ Department of Dermatology, Bangabandhu Sheikh Mujib Medical University,Dhaka, Bangladesh
}

\section{Keywords: \\ Direct immunofluorescence; Hair follicle; Outer root sheath; Pemphigus vulgaris}

\begin{abstract}
Background: Since the outer root sheath of hair follicle is structurally analogous to epidermal keratinocytes, direct immunofluorescence of hair may be an alternate method for monitoring disease activity of pemphigus vulgaris. Our objective was to find the effectiveness of direct immunofluorescence of outer root sheath as an alternate method in monitoring patients with pemphigus vulgaris.

Materials and Methods: Sixteen diagnosed cases of pemphigus vulgaris and in clinical remission for at least three months were enrolled. Skin biopsy sample and five plucked scalp hair samples of each patient were stained with Fluorescein isothiocyanate conjugate.

Results: Hair direct immunofluorescence was positive in $13(81.25 \%)$ patients and skin direct immunofluorescence was also positive in 13 patients $(81.25 \%)$.

Conclusion: Direct immunofluorescence of hair is a simple, specific and non-invasive method, and provides the opportunity to avoid repeated skin biopsies as an appropriate alternative technique for the disease monitoring in patients with pemphigus vulgaris.
\end{abstract}

\section{Correspondence:}

Dr. Mst Sayedatun Nessa, MBBS,MD

Assistant Professor, Department of pathology

Northern International Medical College \& Hospital, Dhaka, Bangladesh 0000-0003-4974-1793

Email: sayedatunnessa78@gmail.com

Reveived : April 30th, 2017 ; Accepted : July 31st, 2017; Published : September 1, 2017

Citation: Sayedatun NM, Kabir AKMN, Barua AR, Paul HK. Role of direct immunofluorescence on outer root sheath of hair follicle in monitoring disease activity of pemphigus vulgaris. J pathol Nep 2017;7:1162-7. doi: 10.3126/jpn.v7i2.17994

Copyright: This is an open-access article distributed under the terms of the Creative Commons Attribution 4.0 International License, which permits unrestricted use, distribution, and reproduction in any medium, provided the original author and source are credited.

\section{INTRODUCTION}

The bullous diseases have a history as old as that of medicine. In the early 1950's, Lever was able to differentiate most of these by using histological criteria. Pemphigus vulgaris (PV) is a very serious skin disease that causes blisters. Pemphigus vulgaris affects $0.1-0.5$ patients per $1,00,000$ population per year ${ }^{1}$ and $0.58-0.80$ per $1,00,000$ person each year in the UK. ${ }^{2}$ Incidence of PV is increasing and the reasons for the changes in incidence are not clearly understood but have implications for identifying causative factors. ${ }^{2}$ Most cases develop in people aged over 50 years. Men and women are equally affected. It is more common 
in certain groups of people - those from the Mediterranean, Indian subcontinent or of Jewish origin.

Large flaccid bullae develop on the oral mucosa, face, scalp, central chest, and inter-triginous zones in older individuals. Oral lesions are the first manifestation in $10 \%$ to $15 \%$ of patients and almost invariably develop during the course of the disease. ${ }^{3}$

Pemphigus is a group of autoimmune blistering disease of skin and mucous membranes which are characterized histologically by intraepidermal blisters due to acantholysis. ${ }^{4}$ Acantholysis was first demonstrated as the characteristic feature of the bullae of pemphigus in 1943. ${ }^{5}$ The autoantibodies in pemphigus are directed against desmosomal proteins both desmoglein (Dsg) 1 and desmoglein (Dsg) 3 resulting in acantholysis, loss of cohesion between keratinocytes and the appearance of bullae. Complement fixation may potentiate the acantholysis. The outer root sheath (ORS) of the hair follicle is structurally analogous to epidermal keratinocytes. ${ }^{6}$ Pemphigus antigens are distributed throughout the ORS and in the dermal bulb matrix cells. ${ }^{6}$ Pemphigus-specific immunofluorescence pattern seen in the skin has been demonstrated in the ORS of a plucked hair follicle. ${ }^{7}$ It characteristically shows the deposition of immunoglobulin G (IgG) with or without complement (C3) in the intercellular region of the epidermis. As the acantholytic process extends into the wall of the hair follicle, immune reactants should also be demonstrable in the follicular wall. Dsg 3 is responsible for anchoring the hair to the follicle. The increase in volume of the target antigen (Dsg 3 and Dsg 1) in the follicular epithelium could be a factor in determining scalp involvement in pemphigus. The gold standard for the demonstration of tissue-bound auto-antibodies is direct immunofluorescence (DIF) of perilesional skin. ${ }^{8}$ DIF pattern of ORS in cases of PV resembles the DIF pattern of the peri-lesional skin. ${ }^{9}$ The sensitivity of hair DIF in PV patients was $100 \%$, similar to that of the cutaneous DIF. ${ }^{10}$ Negative direct immunofluorescence (DIF) has been repeatedly shown to be a better predictor of immunologic remission compared with indirect immunoflurescence. ${ }^{11}$

Obtaining a sample for DIF from oral or skin biopsy is a relatively invasive and unpleasant procedure to the patient. The need for repeating the test until positive results are obtained further complicates the problem; many patients are reluctant to accept it. Hence finding a less-invasive way for collecting a suitable substrate would be of much help. Recently, Schaerer and Trueb et $\mathrm{al}^{7}$ showed that ORS of plucked hair could be used as substrate for DIF in the diagnosis of pemphigus. The value of this method was proven in further studies on patients with untreated or relapsed pemphigus. ${ }^{12}$ Using an alternative substrate for DIF instead of skin or mucosa would be beneficial because it eliminates the need for multiple biopsies in pemphigus patients especially in circumstances of difficult biopsies like
Table 1: Distribution of the study patients by DIF of hair and skin (IgG)(n=16)

\begin{tabular}{lcc}
\hline & $\begin{array}{c}\text { DIF of hair (IgG) } \\
(\mathbf{\%})\end{array}$ & $\begin{array}{c}\text { DIF of skin (IgG) } \\
(\%)\end{array}$ \\
\hline Strong positive(+++) & 0 & 0 \\
$\begin{array}{l}\text { Moderately } \\
\text { positive(++) }\end{array}$ & $1(6.25 \%)$ & $1(6.25 \%)$ \\
\hline Weakly positive $(+)$ & $12(75.0 \%)$ & $12(75.0 \%)$ \\
Negative(-) & $3(18.75 \%)$ & $3(18.75 \%)$
\end{tabular}

Table 2: Comparison between DIF of hair and DIF of skin evaluation for patients with pemphigus vulgaris in clinical remission $(n=16)$

\begin{tabular}{lcc}
\hline \multirow{2}{*}{ DIF of hair } & \multicolumn{2}{c}{ DIF of skin } \\
\cline { 2 - 3 } & $\begin{array}{c}\text { Positive } \\
(\mathrm{n}=13 ; 81.25 \%)\end{array}$ & $\begin{array}{c}\text { Negative } \\
(\mathrm{n}=3 ; 18.25 \%)\end{array}$ \\
\hline $\begin{array}{l}\text { Positive }(\mathrm{n}=13 ; \\
81.25 \%)\end{array}$ & 12 (True positive) & $\begin{array}{c}1 \\
\text { (False positive) }\end{array}$ \\
$\begin{array}{l}\text { Negative }(\mathrm{n}=3 ; \\
18.75 \%)\end{array}$ & 1 & 2 \\
\hline Total (n-16; 100\%) & $\mathbf{1 3}$ & $\mathbf{3 ( 1 8 . 7 5 \% )}$ \\
\hline
\end{tabular}

Table 3: Sensitivity, specificity, accuracy, positive and negative predictive values of DIF of hair evaluation for patients with pemphigus vulgaris in clinical remission

\begin{tabular}{lcc}
\hline Validity test & Percentage & $\mathbf{9 5 \% C I}$ \\
\hline (lower-upper) & 142 & 86 \\
\hline Sensitivity & 0.923 & $(0.79-1.05)$ \\
Specificity & 0.667 & $(0.44-0.90)$ \\
Accuracy & 0.875 & $(0.71-1.04)$ \\
Positive predictive value & 0.923 & $(0.79-1.05)$ \\
\hline Negative predictive value & 0.667 & $(0.44-0.90)$ \\
\hline
\end{tabular}

mucosal areas, or in children. ${ }^{10}$

\section{MATERIALS AND METHODS}

This study was carried out at the Department of pathology, Bangabandhu Sheikh Mujib Medical University (BSMMU), Dhaka and in the Dermatology Department of BSMMU, Dhaka during the period of September'2012 to August' 2014. Approval of the study protocol by the Institutional Review Board of Bangabandhu Sheikh Mujib Medical University, Dhaka was obtained.

A total number of 16 clinically, histologically and immunologically diagnosed cases of pemphigus vulgaris on clinical remission patients were included in this study. The patients were selected from recorded data of DIF on pemphigus vulgaris of Pathology Department of BSMMU and they were followed up in the out-patient Department of Dermatology of BSMMU. The patient was considered to be remission when there was no mucosal or cutaneous lesion, including blisters, erosions, crusts, or vegetative 


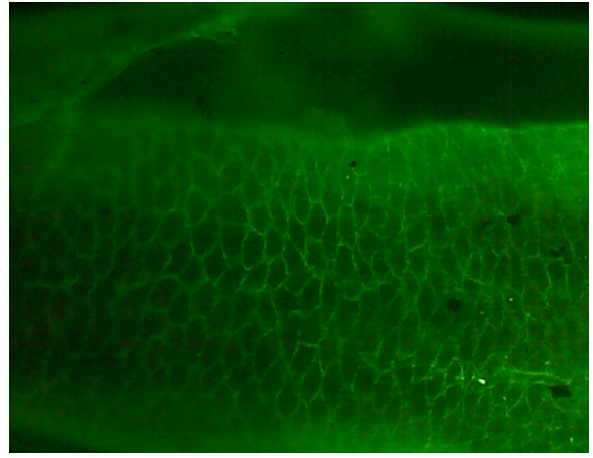

Figure 1: Hair stained by DIF showing intercellular $\operatorname{Ig} G$ deposition in outer root sheath of hair follicle (x400)

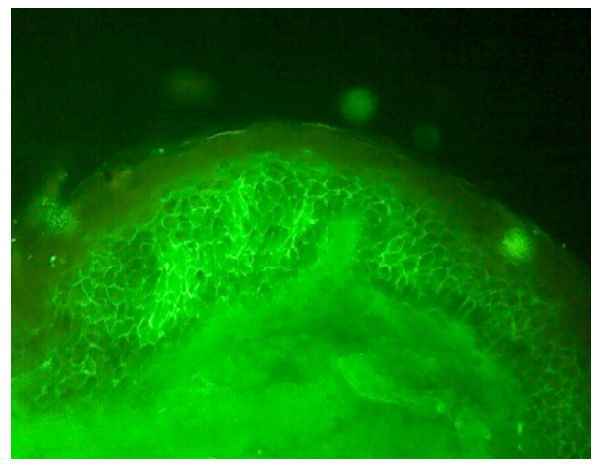

Figure 3: Skin biopsy stained by direct immunofluorescence technique showing lace like deposition of $\operatorname{IgG}$ in squamous intercellular substance of epidermis (x 200)

lesions for at least 3 months while the patient was receiving maintenance therapy with oral prednisolone.

Fulfilling the criteria the patient was selected for collection of specimen. Skin biopsy and plucked scalp hair samples were obtained for direct immunofluorescence stain. Hairs were plucked from the scalp using a rubber tipped artery forceps after applying spray anaesthetic. About five scalp hairs were selected and transported in normal saline and processed for DIF without sectioning. First, the hair samples were taken on a Petri dish and then washed in phosphate buffered saline (PBS) for three times, each wash taking ten minutes. Then the hair samples were placed on a ring glass slide and they were incubated with Fluorescein isothiocyanate conjugated (FITC) rabbit anti-sera against human IgG and complement 3 (Dako, Glostrusp, Denmark) for at least 30 minutes. At the end of the procedure they were washed again in PBS, with three cycles of fifteen minutes each and that time, wash was done over ring glass slide. At that time extra care was taken so that the hair would not float away from the slide. To avoid this, ring slides were placed on a clean plastic box which was kept on slightly slanting position on the table. After washing, the slides were mounted with buffered glycerin mountant and cover slipped and examined under

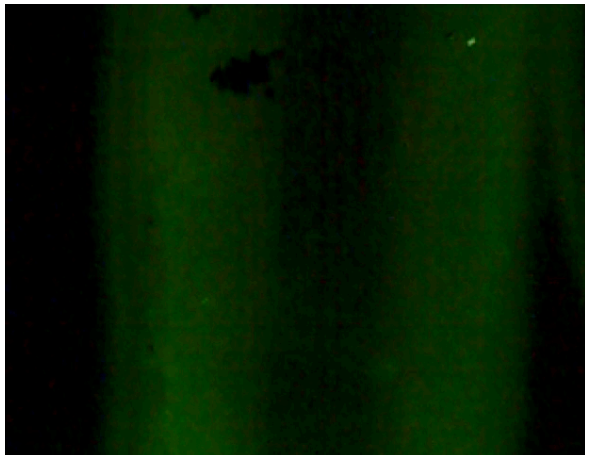

Figure 2: Hair stained by DIF showing no deposition of intercellular IgG in outer root sheath of hair follicle (x 200)

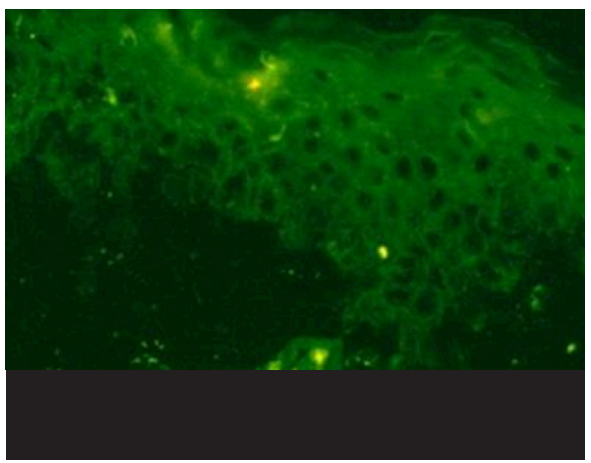

Figure 4: Skin biopsy stained by DIF showing no deposition of $\operatorname{Ig} G$ in intercellular space $(x$ 200)

fluorescence microscope (Olympus BX51)

Full thickness of skin with small subcutaneous tissue as required, by elliptical excisional biopsy specimen was also taken after application of $2 \%$ lidocaine local anesthetic. Specimen was taken in normal saline and after quick freezing; 4-5 micrometer thick sections were cut in the cryostat (Minotome, International Equipment Company, Damon, USA) at $-20^{\circ} \mathrm{C}$ and were taken into glass slides. These were air-dried and kept at $-20^{\circ} \mathrm{C}$ until staining was performed. Staining procedure of skin was same as that of conventional DIF procedure. During this procedure DIF of skin also performed in an equal number of controls. Positive controls were taken from known positive cases and negative controls were taken from other dermatoses.

Result of DIF of skin and ORS of plucked hair was recorded by the same observer and the intensity was arbitrarily graded on a scale from $(+)$ to $(+++)$, or negative $(-)$. Statistical analysis was carried out by using the Statistical Package for Social Sciences (SPSS) version 16. 


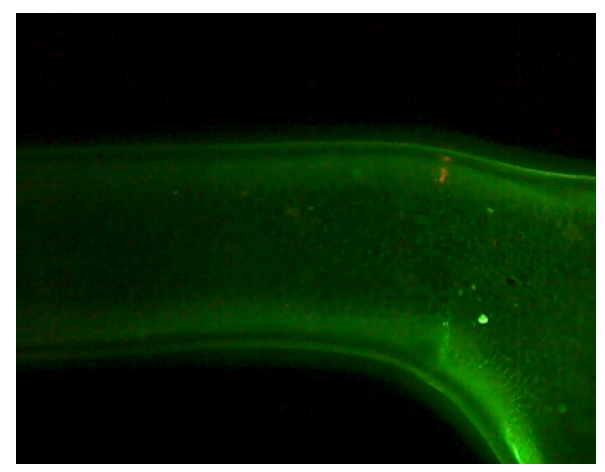

Figure 5: Hair stained by DIF showing intercellular $\operatorname{IgG}(+)$ deposition in outer root sheath of hair follicle (x 200)

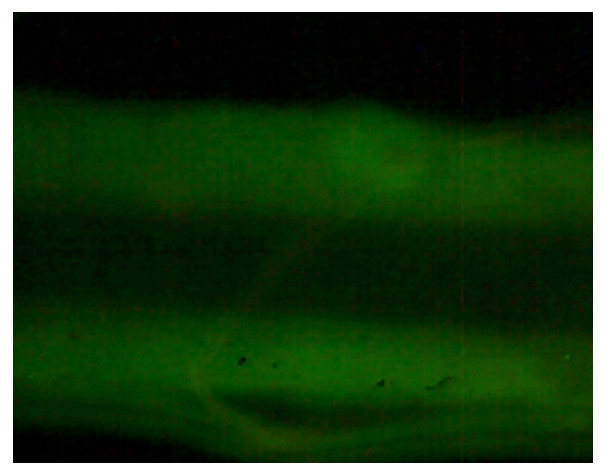

Figure 7: Hair stained by DIF showing no deposition of intercellular $\operatorname{Ig} G$ in outer root sheath of hair follicle (x 200)

\section{RESULTS}

A total of 16 patients clinically, histologically and immunologically diagnosed cases of pemphigus vulgaris on clinical remission were included in this study and scalp hair and perilesional skin biopsy were taken and DIF was done to compare both. A slight preponderance of male patient was observed in the study with male female ratio of 1.7:1. The age distribution of patients varied between 23 to 72 years with mean age of $38.94(\mathrm{SD}=12.28)$ years. The main age group involved was fourth decade. Seven cases (43.75) belonged to this group.

On direct immunofluorescence test of hair (IgG), among the 16 patients 13 showed intercellular IgG deposition in outer root sheath of hair follicle DIF (fig.1). Three cases showed no such IgG deposition in ORS of hair follicle (fig. 2). On DIF test of skin (IgG), among the 16 patients 13 showed lace like deposition of IgG in squamous intercellular space of epidermis (fig. 3). Similarly 3 cases showed no such $\operatorname{IgG}$ deposits (fig. 4). Twelve patients were positive in both hair and skin DIF. There was one case with false positive as ORS of hair follicle DIF was positive (fig.5) but skin DIF was

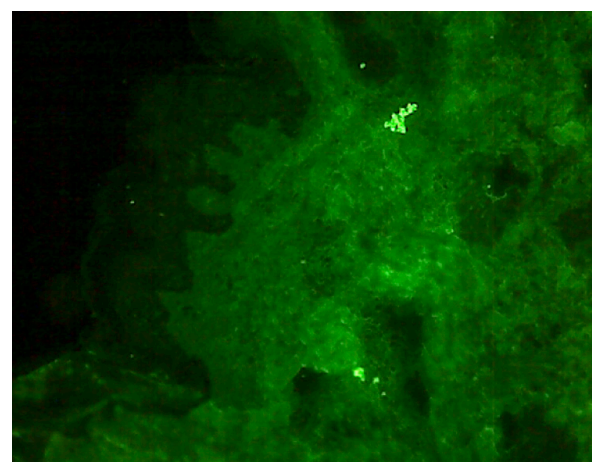

Figure 6: Skin biopsy stained by DIF showing no deposition of IgG in intercellular space ( $x$ 200)

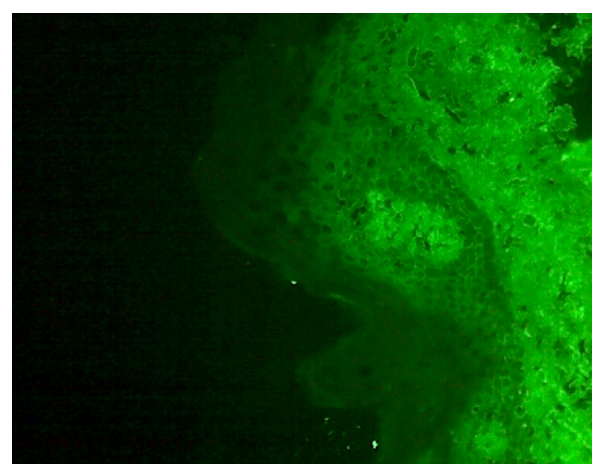

Figure 8: Skin biopsy stained by direct immunofluorescence technique showing lace like deposition of IgG in squamous intercellular substance of epidermis (DIFx 200)

negative (fig.6). One case showed false negative result as ORS of hair follicle was negative (fig.7) but positive on skin DIF (fig.8). Two patients were negative on both hair and skin DIF test. On DIF of skin for C3, 3(18.25\%) patients had weakly positive and $13(81.25 \%)$ had negative result. DIF of hair for $\mathrm{C} 3$ of the study patients showed negative result in all $(100 \%)$ cases.

Table 1 shows DIF of skin (IgG) and hair (IgG) of the study patients, it was observed that three fourth $(75.0 \%)$ patients had weakly positive, one (6.25\%) had moderately positive and three $(18.75 \%)$ had negative on DIF of both skin $(\operatorname{IgG})$ and hair( $\operatorname{IgG})$.

Conventional DIF is known as the best method for assessing immunologic remission in PV and was used as the gold standard in statistical analysis with which hair DIF was compared. Out of 16 patients with pemphigus in clinical remission 02 showed true negative result on DIF e.g. DIF was negative both in skin and hair. Twelve patients were true positive e.g. DIF was positive in both skin and hair. One of them was false positive e.g. DIF was positive in hair but negative in skin. One of them was false negative as DIF 
was negative in hair but positive in skin. Table 2 shows the distribution of frequency of hair and conventional direct immunofluorescence in patient with pemphigus in clinical remission.

Direct immunofluorescence of hair evaluation for patients with pemphigus vulgaris in clinical remission is described in table 2. There were 12 true positive cases, 1 false positive case, 1 false negative case and 2 true negative cases in identification by DIF of skin. The results of the interpreter analysis are Kappa $=0.590$ with $\mathrm{p}<0.05(0.018)$ with moderate agreement and statistically significant.

Sensitivity of hair DIF (i.e. the percentage of patients with positive conventional DIF whose hair DIF was positive), specificity of hair DIF (i.e. the percentage of patients with negative conventional DIF whose hair DIF was negative), positive predictive value of hair DIF (ie, the percentage of patients with positive hair DIF whose conventional DIF was also positive), and negative predictive value of hair DIF (i.e. the percentage of patients with negative hair DIF whose conventional DIF was also negative) were calculated (Table 3). Positive predictive value enables us to tell how likely it is that the patient is not in immunologic remission if his/ her hair DIF is positive. Negative predictive value enable us to tell how likely it is that the patient is in immunologic remission if his/her hair DIF is negative.

The validity of DIF of skin evaluation for patients with pemphigus vulgaris in clinical remission was correlated. Table 3 shows hair DIF had a sensitivity of $0.923(95 \%$ confidence interval [CI] 0.79-1.05), a specificity of 0.667 (95\% CI 0.44- 0.90), an accuracy of 0.875 (95\% CI 0.71 1.04 ), a positive predictive value of 0.923 (95\% CI 0.79 $1.05)$ and a negative predictive value of 0.667 (95\% CI 0.44- 0.90).

\section{DISCUSSION}

The goal of therapy in pemphigus is to achieve clinical and immunological remission. Clinical remission is achieved in most patients with immunosuppressive agents. The most difficult management decision is how to maintain remission with the least number of medications. Although many pemphigus authorities rely on clinical status to discontinue treatment, assessing immunologic remission by DIF has its own advocates. Actually, many studies have shown that the rate of relapse in patients in clinical remission after discontinuing therapy was higher when DIF was positive. ${ }^{13,14}$ So the treatment should be continued in the patients with positive DIF results. On the other hand, repeated negative DIF during remission is a possible marker for apparent cure of the disease, and treatment can be stopped in such group of patients. ${ }^{11}$ Patients in remission may refuse to undergo repeated skin biopsies. The present study was undertaken with the goal to find out an alternate reliable source of substrate for DIF test in monitoring of pemphigus vulgaris.
In this study the main age group involved was fourth decade. Seven cases (43.75) belong to this group. The age range was 23 to 72 years with mean age of $38.94(\mathrm{SD}=12.28)$ years. Male female ratio was 1.7:1. These finding differ from Raoet $\mathrm{al}^{15}$ and Daneshpazhooh et $\mathrm{al}^{16}$ where mean age was around 45 and female was more than male. This difference may be due to small sample size.

In this study on direct immunofluorescence test of hair (IgG), among the 16 patients $13(81.25 \%)$ were positive and $3(18.75 \%)$ were negative. On DIF test of skin (IgG), among the 16 patients $13(81.25 \%)$ were positive and 3 $(18.75 \%)$ were negative. Twelve patients were positive in both hair and skin DIF considered as true positive. There was one false positive case whose hair DIF was positive but skin DIF was negative. One patient was false negative who was negative on hair DIF but positive on skin DIF. Two patients were true negative i.e. negative on both hair and skin DIF test. Schaerer and Trueb ${ }^{7}$ first demonstrated the practicability of using DIF on plucked hair, and they were able to demonstrate the presence of the pemphigus specific DIF pattern in the ORS of all of their $15(100 \%)$ pemphigus patients. Rao et $a l^{15}$ found $85 \%$ DIF positivity in the ORS of plucked anagen hair. Daneshpazhooh et al ${ }^{16}$ reported $91 \%$ DIF positivity in ORS of plucked hair from 110 patients with PV. In our study, the DIF pattern in the ORS of scalp hair was $81.25 \%$ which is almost similar with other studies.

Danesgpazhoohet $\mathrm{a}^{12}$ found the sensitivity of hair DIF in 110 patients with untreated PV and positive DIF on oral mucosal biopsy to be 0.91 . Rao $\mathrm{R}$ et al ${ }^{15}$ found 0.85 sensitivity of hair DIF in active pemphigus. In another study, Daneshpazhooh et al ${ }^{16}$ described sensitivity of hair DIF as 0.79 and a specificity of 0.48 . In this study we compared plucked hair DIF and conventional DIF to assess immunologic remission. What is important in this context is not to stop steroids while the patient is not yet in immunologic remission. In other words, false negatives have to be minimal. Therefore the sensitivity is important in this setting to avoid premature discontinuation of the drug and relapse. In this study we found only one case having false negative result. The sensitivity of the DIF of plucked hair was 0.92 . Although relatively high, the figure was lower than reported by Schaerer and Trueb ${ }^{7}$ which was $1(100 \%)$. Daneshpazhooh et al ${ }^{12}$ found sensitivity of plucked hair DIF as 0.91 which was comparable with present study. In our study sensitivity of plucked hair DIF and skin biopsy taken from the patients who were in clinical remission was same. In the study of Daneshpazhooh et $\mathrm{al}^{16}$, positive predictive value of plucked hair DIF was 0.61 and negative predictive value was 0.68 . In present study positive predictive value is 0.92 and negative predictive value is 0.66 . All these statistical data are comparable with recent studies and significant enough.

Pemphigus vulgaris can be controlled with treatment in most instances but difficult to maintain control with the 
least medication. Long-term remission of all therapy has been reported to occur in $10-75 \%$ of the patients. ${ }^{17}$ The differentiation between drug induced clinical control of the disease in the presence of autoantibodies, from immunological inactivity can help us to detect the subgroup of patients who maintain clinical remission after discontinuation of treatment. However immunological inactivity of the disease may also be drug induced and relapse may occur after discontinuation of therapy even in these patients. Furthermore, immunological remission of disease after discontinuation of drugs does not necessarily indicate a cure. According to a previous report, many patients with negative DIF findings $(73.3 \%)$ remained in clinical remission after discontinuation of treatment. All patients with positive DIF findings during remission had a relapse within 3 months after discontinuation of treatment. ${ }^{13}$

Thus, DIF findings may be indicative of either further regression or eminent exacerbation. Therapy should be continued in patients with positive DIF results to avoid relapse. ${ }^{14}$ On the other hand, treatment can be stopped in those groups of patients showing repeated negative DIF during remission which indicates apparent cure of the disease. David et al ${ }^{11}$ studied 24 PV patients and treatment was discontinued in seven patients with repeated negative results of DIF. One out of seven patients (14\%) relapsed in a 14 months follow-up period.

Patients need repeated DIF testing during diagnosis and in the follow up after clinical remission to be sure of immunological remission. DIF of skin is still the gold standard for the diagnosis of pemphigus vulgaris. But patients in remission stage may don't agree to do repeat skin biopsies as it is an invasive procedure. DIF of hair may be an ideal choice of substrate as it is a simple and non-invasive test with an equal degree of sensitivity and specificity.

\section{CONCLUSION}

This study proved the value of plucked hair as an appropriate substrate for DIF for the disease monitoring of PV. In addition to its relatively high sensitivity DIF of hair is a simple, specific and noninvasive test, and provides the opportunity to avoid repeated skin biopsies in patients with pemphigus vulgaris.

\section{ACKNOWLEDGMENTS}

Our sincere gratitude all the patients whose sample has helped conduct us this study. We would also like to thank all the faculties of department of pathology and dermatology and technical staffs for their support. My deepest regards and sincerest gratitude to respected teacher and guide Professor Ashim Ranjan Barua, department of pathology, BSMMU, Dhaka, for all his able guidance and whole hearted co-operation in making this study a success.

\section{Conflict of interest: None}

\section{REFERENCES}

1. Leena, Vijaya B, Manjunath GV, Sunila. A clinicopathological study of 22 cases of pemphigus. J Clin Diag Res 2010;4:2446-2451.

2. Langan SM, Smeeth L, Hubbard R et al. Bullous pemphigoid and pemphigus vulgaris- incidence and mortality in the UK. Br Med J 2008;337:180. Crossref

3. Younus J, Ahmed AR. The relationship of pemphigous to neoplasia. J Am Acad Dermatol 1990;23:498. Crossref

4. David E Elder. Lever's histopathology of the skin.10th ED. Wolters kluwer: Philadelphia; 2004.1408pp

5. Cohen LM, Skopicki DK, Harrist TJ, Clark WH Jr. Non infectious vesiculobullous and vesiculopustular diseases. In : Elder D, Elenitras R,Jawarsky C, Johnson Jr. B, (eds). Levers histopathology of skin.8th ed. Lipincott Raven:Philadelphia; 1997. pp209-387.

6. Wilson CL, Dean D, Wojnarowska F. Pemphigus and the terminal hair follicle. J CutanPathol1991;18:428-43. Crossref

7. Schaerer L, Trueb RM. Direct immunofluorescence of plucked hair in pemphigus. Arch Dermatol 2003;139:228-229. Crossref

8. Mahalingam M. Follicular acantholysis: a subtle clue to the early diagnosis of Pemphigus vulgaris. Am J Dermatopathol 2005;27:237239. Crossref

9. Kumaresan M, Rai R, SandhyaV. Immunofluorescence of the outer root sheath: an aid todiagnosis in pemphigus. British ClinExpDermatol2010; 36:298-301.

10. Alexandru A, Zurac S, Salavastru CM, et al. Direct immunofluorescence on hair follicles present and future perspectives. Am J Dermatopathol 2013;34:472-476. Crossref

11. David M, Weissman V, Ben A, et al. The usefulness of immunofluorescent tests in pemphigus patients in clinical remission. Br J Dermatol 1989; 120: 391-4. Crossref

12. Daneshpazhooh M, Asgari M, Naraghi ZS, et al. A study on plucked hair as a substrate for direct immunofluorescence in pemphigus vulgaris. J Eur Acad Dermatol venereol 2009;23:129-131. Crossref

13. Ratnam KV, Pang BK. Pemphigus in remission: value of negative immunofluorescence in management.J Am AcadDermatol 1994;30: 547-550. Crossref

14. Balighi K, Taheri A, Mansoori P, Chams C. Value of direct immunofluorescence in predicting remission in pemphigu vulgaris. Int J Dermatol 2006;45:1308-11. Crossref

15. Rao R, Dasari k, Shenoi S, Balachandram C. Demonstration of pemphigus specific immunofluorescence pattern by direct immunofluorescence of plucked hair. Int J Dermatol2009; 48:11871189. Crossref

16. Daneshpazhooh M, Zahara S, Naraghi ZS,et al.Direct immunofluorescence of plucked hair for evaluation of immunologic remission of pemphigous vulgaris. J Am Acad dermatol 2011;65:173177. Crossref

17. Mourellou O, Chaidemenos GC, Koussidou T,et al. The treatment of pemphigus vulgaris. Experience with 48 patients seen over an 11 year perod. Br J Dermatol 1995;133:83-87. Crossref 